doi: https://doi.org/10.15407/dopovidi2018.07.020

UDC 512.542

\title{
V.S. Yashchuk
}

Oles Honchar Dnipro National University

E-mail: Viktoriia.Yashchuk@i.ua

\section{Leibniz algebras of dimension 3 over finite fields}

Presented by Corresponding Member of the NAS of Ukraine V.P. Motornyi

The first thing in the study of all types of algebras is the description of algebras having small dimensions. Unlike the simpler cases of 1- and 2-dimensional Leibniz algebras, the structure of 3-dimensional Leibniz algebras is more complicated. We consider the structure of Leibniz algebras of dimension 3 over a finite field. In some cases, the structure of the algebra essentially depends on the characteristic of the field. In others, it depends on the solvability of specific equations in the field, and so on.

Keyzords: Leibniz algebra, ideal, factor-algebra, nilpotent Leibniz algebra.

Let $\mathrm{L}$ be an algebra over a field $F$ with the binary operations + and [ $\cdot \cdot]$. Then $L$ is called a Leibniz algebra (more precisely, a left Leibniz algebra), if it satisfies the (left) Leibniz identity

$[[a, b], c]=[a,[b, c]]-[b,[a, c]]$ for all $a, b, c \in L$.

We will also use another form of this identity:

$$
[a,[b, c]]=[[a, b], c]+[b,[a, c]] .
$$

Leibniz algebras appeared first in the papers of A.M. Bloh [1-3], in which he called them the D-algebras. However, in that time, these works were not in demand, and they have not been properly developed. Only after two decades, a real interest in Leibniz algebras rose. It happened thanks to the work of J.-L. Loday [4] (see also [5, Section 10.6]), who "rediscovered" these algebras and used the term Leibniz algebras, since it was Gottfried Wilhelm Leibniz who discovered and proved the Leibniz rule for the differentiation of functions.

Let $L$ be a Leibniz algebra over a field $F$. If $A, B$ are subspaces of $L$, then $[A, B]$ will denote a subspace generated by all elements $[a, b]$ where $a \in A, b \in B$. As usual, a subspace $A$ of $L$ is called a subalgebra of $L$, if $[x, y] \in A$ for every $x, y \in A$. It follows that $[A, A] \leqslant A$.

Let $L$ be a Leibniz algebra over a field $F$, and let $M$ be a non-empty subset of $L$. Then $\langle M\rangle$ denotes the subalgebra of $L$ generated by $M$.

A subalgebra $A$ of $L$ is called a left (respectively, right) ideal of $L$, if $[y, x] \in A$ (respectively, $[x, y] \in A$ ) for every $x \in A, y \in L$. In other words, if $A$ is a left (respectively, right) ideal, then $[L$, $A] \leqslant A$ (respectively, $[A, L] \leqslant A$ ).

(C) V.S. Yashchuk, 2018 
A subalgebra $A$ of $L$ is called an ideal of $L$ (more precisely, two-sided ideal), if it is both a left ideal and a right ideal, i. e., $[y, x],[x, y] \in A$ for every $x \in A, y \in L$.

If $A$ is an ideal of $L$, we can consider a factor-algebra $L / A$. It is not hard to see that this factoralgebra also is a Leibniz algebra.

As usual, a Leibniz algebra $L$ is called abelian, if $[x, y]=0$ for all elements $x, y \in L$. In an Abelian Leibniz algebra, every subspace is a subalgebra and an ideal.

Denote, by Leib $(L)$, the subspace generated by the elements $[a, a], a \in L$. It is possible to prove that $\operatorname{Leib}(L)$ is an ideal of $L$ such that $L / \operatorname{Leib}(L)$ is a Lie algebra. Conversely, if $H$ is an ideal of $L$ such that $L / H$ is a Lie algebra, then $\operatorname{Leib}(L) \leqslant H$.

The ideal $\operatorname{Leib}(L)$ is called a Leibniz kernel of the algebra $L$.

We note a following important property of the Leibniz kernel:

$[[a, a], x]=[a,[a, x]]-[a,[a, x]]=0$.

This property shows that $\operatorname{Leib}(L)$ is an abelian subalgebra of $L$.

As usual, we say that a Leibniz algebra $L$ is finite-dimensional, if the dimension of $L$ as a vector space over $F$ is finite.

The first step in the study of all types of algebras is the description of algebras having small dimensions.

If $\operatorname{dim}_{\mathrm{F}}(L)=1$, then $L$ is an abelian Lie algebra, i. e., is $L=F a$ for some element $a$ and $[a, a]=0$.

If $\operatorname{dim}_{\mathrm{F}}(L)=2$ and $L$ is not a Lie algebra, then there are the following two non-isomorphic Leibniz algebras:

and

$$
L_{1}=F a+F b,[a, a]=b,[b, a]=[a, b]=[b, b]=0,
$$

$$
L_{2}=F c+F d,[c, c]=[c, d]=d,[d, c]=[d, d]=0
$$

(see, e. g., survey [6]). The structure of 3-dimensional Leibniz algebras is more complicated. The study of Leibniz algebras, having dimension 3, has been conducted in papers [7-10] for the fields of characteristic 0 , moreover for a field $\mathrm{C}$ of complex numbers or an algebraically closed field of characteristic 0 . We consider the opposite situation, where the structure of Leibniz algebras of dimension 3 over a finite field should be described. As we will see later, the situation here is much more diverse. In some cases, the structure of the algebra essentially depends on the characteristic of the field, in others on the solvability of specific equations in the field, and so on. We will see that the Leibniz algebras of dimension 3 are soluble. Therefore, a first natural step of our study is a consideration of nilpotent algebras.

Let L be a Leibniz algebra. Define the lower central series

$$
L=\gamma_{1}(L) \geqslant \gamma_{2}(L) \geqslant \ldots \gamma_{\alpha}(L) \geqslant \gamma_{\alpha+1}(L) \geqslant \ldots \gamma_{\delta}(L)
$$

of $L$ by the following rule: $\gamma_{1}(L)=L, \gamma_{2}(L)=[L, L]$, and, recursively $\gamma_{\alpha+1}(L)=\left[L, \gamma_{\alpha}(L)\right]$ for all ordinals $\alpha$ and $\gamma_{\lambda}(L)=\cap_{\mu<\lambda} \gamma_{\mu}(L)$. It is possible to show that every term of this series is an ideal of $L$. The last term $\gamma_{\delta}(L)$ is called the lower hypocenter of $L$. We have $\gamma_{\delta}(L)=\left[L, \gamma_{\delta}(L)\right]$.

If $\alpha=k$ is a positive integer, then $\gamma_{\mathrm{k}}(L)=[L,[L,[L, \ldots] \ldots]]$. Note the following useful properties of subalgebras and ideals. 
A Leibniz algebra $L$ is called nilpotent, if there exists a positive integer $k$ such that $\gamma_{k}(L)=\langle 0\rangle$. More precisely, $L$ is said to be nilpotent of the nilpotency class $\mathbf{c}$, if $\gamma_{\mathbf{c}+1}(L)=\langle 0\rangle$, but $\gamma_{\mathbf{c}}(L) \neq\langle 0\rangle$. We denote, by $\operatorname{ncl}(L)$, the nilpotency class of $L$.

The left (respectively, right) center $\zeta^{\text {left }}(\mathrm{L})$ (respectively, $\zeta^{\text {right }}(\mathrm{L})$ ) of a Leibniz algebra $\mathrm{L}$ is defined by the rule:

$$
\zeta^{\operatorname{left}}(L)=\{x \in L \mid[x, y]=0 \text { for each element } y \in L\}
$$

(respectively,

$$
\left.\zeta^{\text {right }}(L)=\{x \in L \mid[y, x]=0 \text { for each element } y \in L\}\right) .
$$

It is not hard to prove that the left center of $L$ is an ideal, but this is not true for the right center. Moreover, the last equality shows that $\operatorname{Leib}(L) \leqslant \zeta^{\text {left }}(L)$, so that $L / \zeta^{\text {left }}(L)$ is a Lie algebra. The right center is an subalgebra of $L$. In general, the left and right centers are different; they even may have different dimensions. We will construct now a following examples [11].

Of course, we will consider a case where $L$ is not a Lie algebra.

Nilpotent Leibniz algebra of dimension 3. In this section, we will suppose that $L$ is nilpotent. Since $\operatorname{ncl}(L) \leqslant \operatorname{dim}_{\mathrm{F}}(L)$, we have $\operatorname{ncl}(L) \leqslant 3$.

Let $L$ be a Leibniz algebra. The intersection of a maximal subgroup of $L$ is called the Frattini subalgebra of $L$ and denoted by $\operatorname{Frat}(L)$. If $L$ does not include maximal subalgebras, then we put $L=\operatorname{Frat}(L)$.

We will need the following important property of Frattini subalgebras.

Proposition 1. Let L be a finite-dimensional Leibniz algebra over a field F. If $L$ is nilpotent, then $[L, L]=\operatorname{Frat}(L)$.

Indeed, since $L$ is nilpotent, every maximal subalgebra of $L$ is an ideal [12, Lemma 2.2], so we can apply Proposition 7 of paper [6].

Theorem 1. Let $L$ be a nilpotent Leibniz algebra over a field $F$. If $L$ is not a Lie algebra and $\operatorname{ncl}(L)=3=\operatorname{dim}_{\mathrm{F}}(L)$, then $L$ has a basis $\{a, b, c\}$ such that $[a, a]=b,[a, b]=c,[c, a]=[a, c]=$ $=[c, b]=[b, c]=[b, b]=[c, c]=0$. Moreover, $\operatorname{Leib}(L)=\zeta^{\text {left }}(L)=[L, L]=F b \oplus F c$, $\zeta^{\text {right }}(L)=$ $=\zeta(L)=\gamma_{3}(L)=F$ c. In particular, $L$ is a nilpotent cyclic Leibniz algebra.

Further, the relation $L=A \oplus \mathrm{B}$ means that $L$ is a direct sum of the subspaces $A$ and $B$ or the subalgebras $A$ and $B$. If $L=A \oplus B, A$ is an ideal of $L$, and $B$ is a subalgebra of $L$, then we will say that $L$ is a semidirect sum of $A$ and $B$ and use the symbol $L=A \dashv B$.

Theorem 2. Let $L$ be a nilpotent Leibniz algebra over a field $F$. Suppose that $L$ is not a Lie algebra, $\operatorname{dim}_{F}(L)=3, \operatorname{ncl}(L)=2$ and $L$ has an element $b \notin \gamma_{2}(L)$ such that $[b, b]=0$. Then $L$ is an algebra of one of the following types:

I. $L=A \oplus B$, where $A, B$ are the ideals, $B=F b,[b, b]=0, A=F a \oplus F c$ is a cyclic nilpotent subalgebra, $[a, a]=c,[c, a]=[a, c]=[c, c]=0$. Moreover, $\operatorname{Leib}(L)=[L, L]=F c, \zeta^{\text {left }}(L)=\zeta^{\text {right }}(L)=$ $=\zeta(L)=F b \oplus F c$.

II. $L=A \dashv B$, where $A=F a \oplus F c$ is a cyclic nilpotent subalgebra, $[a, a]=c,[c, a]=[a, c]=$ $=[c, c]=0, B$ is an abelian subalgebra, $B=F b,[b, b]=0$, and $[a, b]=c,[b, a]=0=[b, c]=[c, b]$. Moreover, $\operatorname{Leib}(L)=[L, L]=\zeta^{\text {right }}(L)=\zeta(L)=F c$, $\zeta^{\text {left }}(L)=F b \oplus F c$.

III. $L=A \dashv B$, where $A=F a \oplus F c$ is a cyclic nilpotent subalgebra, $[a, a]=c,[c, a]=[a, c]=$ $=[c, c]=0, B$ is an abelian subalgebra, $B=F b,[b, b]=0$, and $[a, b]=c,[b, a]=\gamma c, \gamma \neq 0,[b, c]=[c$, $b]=0$. Moreover, $\operatorname{Leib}(L)=[L, L]=\zeta^{\text {left }}(L)=\zeta^{\text {right }}(L)=\zeta(L)=F c$. 
Theorem 3. Let $L$ be a nilpotent Leibniz algebra over a field $F$. Suppose that $L$ is not a Lie algebra, $\operatorname{dim}_{\mathrm{F}}(L)=3, \operatorname{ncl}(L)=2$, and $[d, d] \neq 0$ for each element $d \notin \gamma_{2}(L)$ such that $[b, b]=0$. Then $L$ is an algebra of one of the following types:

I. $L=A+B$, where $A, B$ are the nilpotent ideals, $A=\langle a\rangle, B=\langle b\rangle, A \cap B=\zeta(L)=F c,[a, a]=$ $=[b, b]=c,[c, a]=[a, c]=[c, c]=[c, b]=[b, c]=[a, b]=[b, a]=0$. Moreover, Leib $(L)=[L, L]=$ $=\zeta^{\text {left }}(L)=\zeta^{\text {right }}(L)=\zeta(L)=F c$, $\operatorname{char}(F) \neq 2$, and the equation $x^{2}+1=0$ has no solution in $F$.

II. $L=A+B$, where $A, B$ are the nilpotent ideals, $A=\langle a\rangle, B=\langle b\rangle, A \cap B=\zeta(L)=F c,[a, a]=c$, $[b, b]=\rho c$, where $\rho$ is a primitive root of the identity of degree $|F|-1,[c, a]=[a, c]=[c, c]=[c, b]=$ $=[b, c]=[a, b]=[b, a]=0$. Moreover, $\operatorname{Leib}(L)=[L, L]=\zeta^{\operatorname{left}}(L)=\zeta^{\mathrm{right}}(L)=\zeta(L)=F c, \operatorname{char}(F) \neq 2$.

III. $L=A+B$, where $A, B$ are the nilpotent ideals, $A=\langle a\rangle, B=\langle b\rangle, A \cap B=\zeta(L)=F c,[a, a]=$ $=c=[a, b],[b, b]=\eta c,[c, a]=[a, c]=[c, c]=[c, b]=[b, c]=[b, a]=0$. Moreover, $\operatorname{Leib}(L)=[L, L]=$ $=\zeta^{\text {left }}(L)=\zeta^{\text {right }}(L)=\zeta(L)=F c$, and a polynomial $X^{2}+X+\eta$ has no roots in a field $F$.

Non-nilpotent Leibniz algebra of dimension 3 with one-dimensional Leibniz kernel. The next step is a consideration of a case where $L$ is non-nilpotent. We will consider Leibniz algebras of dimension 3, which are not Lie algebras. It follows that $\operatorname{Leib}(L) \neq\langle 0\rangle$. Since $\operatorname{Leib}(L)$ is an abelian ideal, $L \neq \operatorname{Leib}(L)$. Hence, for $\operatorname{Leib}(L)$, we have only two possibility: $\operatorname{dim}_{\mathrm{F}}(\operatorname{Leib}(L))=1$, $\operatorname{dim}_{\mathrm{F}}(\operatorname{Leib}(L))=2$.

In this section, we consider the case where $\operatorname{dim}_{\mathrm{F}}(\operatorname{Leib}(L))=1$, so that $\operatorname{dim}_{\mathrm{F}}(L / \operatorname{Leib}(L))=2$.

Theorem 4. Let $L$ be a non-nilpotent Leibniz algebra over a field $F$. Suppose that $L$ is not a Lie algebra, $\operatorname{dim}_{\mathrm{F}}(L)=3$ and $\operatorname{dim}_{\mathrm{F}}(\operatorname{Leib}(L))=1$. Then $L$ is an algebra of one of the following types:

I. $L=A \oplus B$, where $A, B$ are the ideals, $B=F b,[b, b]=0, A$ is a cyclic subalgebra, $A=F a \oplus F c$, where $[a, a]=c=[a, c],[c, a]=[c, c]=[c, b]=[b, c]=[a, b]=[b, a]=0$. Moreover, $\operatorname{Leib}(L)=[L$, $L]=F c$, $\zeta^{\text {left }}(L)=F b \oplus F c$, $\zeta^{\operatorname{right}}(L)=\zeta(L)=F b$.

II. $L=A \dashv B$, where $B=F b,[b, b]=0, A=F a \oplus F c$ is a cyclic subalgebra, $[a, a]=c=[a, c],[a$, $b]=c,[c, a]=[c, c]=[c, b]=[b, c]=[b, a]=0$. Moreover, $\operatorname{Leib}(L)=[L, L]=F c, \zeta^{\text {left }}(L)=F b \oplus F c$, $\zeta(L)=\zeta^{\mathrm{right}}(L)=\langle 0\rangle$.

III. $L=A \dashv B$, where $B=F b,[b, b]=0, A=F a \oplus F c$ is a cyclic subalgebra, $[a, a]=c=[a, c],[b$, $a]=[b, c]=c,[c, a]=[c, c]=[c, b]=[a, b]=0$. Moreover, $\operatorname{Leib}(L)=[L, L]=\zeta^{\text {left }}(L)=F c, \zeta^{\text {right }}(L)=$ $=F b, \zeta(L)=\langle 0\rangle$.

IV. $L=A \dashv B$, where $B=F b,[b, b]=0, A=F a \oplus F c$ is a cyclic subalgebra, $[a, a]=c=[a, c]$, $[a, b]=a=-[b, a],[b, c]=-2 c,[c, a]=[c, c]=[c, b]=0$. Moreover, $\operatorname{Leib}(L)=[L, L]=\zeta^{\text {left }}(L)=F c$, $\zeta^{\text {right }}(L)=\zeta(L)=\langle 0\rangle$.

V. $L=A \dashv B$, where $B=F b,[b, b]=0, A=F a \oplus F c$ is a cyclic subalgebra, $[a, a]=c,[a, c]=$ $=0,[a, b]=a+\gamma c, \gamma \in F,[b, a]=-a+\gamma c,[b, c]=-2 c,[c, a]=[c, c]=[c, b]=0$. Moreover, Leib $(L)=$ $=[L, L]=\zeta^{\text {left }}(L)=F c, \zeta^{\text {right }}(L)=\zeta(L)=\langle 0\rangle$ whenever $\operatorname{char}(F) \neq 2$ and $\zeta^{\text {right }}(L)=\zeta(L)=F c$ whenever $\operatorname{char}(F)=2$.

Non-nilpotent cyclic Leibniz algebra of dimension 3. The next step is a consideration of a case where $L$ is non-nilpotent, and $\operatorname{dim}_{\mathrm{F}}(\operatorname{Leib}(L))=2$. Here, there appear two variants: $L$ is a cyclic algebra and $L$ is a non-cyclic algebra. In this section, we will consider a case where a Leibniz algebra of dimension 3 is cyclic.

Theorem 5. Let L be a non-nilpotent cyclic Leibniz algebra of dimension 3 over a field $F$. Then $L$ is an algebra of one of the following types: 
I. $L=D \dashv A$, where $D=F d,[d, d]=0, A=F a \oplus F c$ is a cyclic nilpotent subalgebra, $[a, a]=c,[a$, $c]=0,[a, d]=\delta d, 0 \neq \delta \in F,[c, a]=[c, c]=[c, d]=[d, c]=[d, a]=0$. Moreover, $\operatorname{Leib}(L)=[L, L]=$ $\zeta^{\text {left }}(L)=F d \oplus F c, \zeta(L)=\zeta^{\text {right }}(L)=F c$.

II. $L=D \dashv B$, where $B=F b,[b, b]=0, D=F d \oplus F c$ is an abelian subalgebra, $[d, d]=[d, c]=$ $=[c, d]=[c, c]=0,[b, c]=d,[b, d]=\gamma d+\delta d, 0 \neq \gamma, \delta \in F,[c, b]=[d, b]=0$. Moreover, $\operatorname{Leib}(L)=$ $=[L, L]=\zeta^{\operatorname{left}}(L)=F d \oplus F c, \zeta^{\mathrm{right}}(L)=F b, \zeta(L)=\langle 0\rangle$.

Non-nilpotent Leibniz algebra of dimension 3 with two-dimensional Leibniz kernel. The last case of our consideration is the case where $L$ is non-nilpotent, non-cyclic, and $\operatorname{dim}_{\mathrm{F}}(\operatorname{Leib}(L))=2$. Then $\operatorname{dim}_{\mathrm{F}}(L / \operatorname{Leib}(L))=1$. In particular, $L / \operatorname{Leib}(L)$ is Abelian.

Theorem 6. Let $L$ be a non-nilpotent non-cyclic Leibniz algebra of dimension 3 over a field $F$. Suppose that $L$ is a not Lie algebra and $\operatorname{dim}_{\mathrm{F}}(\operatorname{Leib}(L))=2$. Then $L$ is an algebra of one of the following types:

I. $L=A \dashv D$, where $D=F d,[d, d]=0, A=F a \oplus F c$ is a cyclic subalgebra, $[a, a]=c=[a, c]$, $[a, d]=d,[c, a]=[c, c]=[c, d]=[d, c]=[d, a]=0$. Moreover, $\operatorname{Leib}(L)=[L, L]=\zeta^{\mathrm{left}}(L)=F d \oplus F c$, $\zeta(L)=\zeta^{\mathrm{right}}(L)=\langle 0\rangle$.

II. Char $(F) \neq 2, L=A \dashv D$, where $D=F d,[d, d]=0, A=F a \oplus F c$ is a cyclic subalgebra, $[a, a]=$ $=c=[a, c],[a, d]=c+2 d,[c, a]=[c, c]=[c, d]=[d, c]=[d, a]=0$. Moreover, $\operatorname{Leib}(L)=[L, L]=$ $=\zeta^{\text {left }}(L)=F d \oplus F c, \zeta(L)=\zeta^{\text {right }}(L)=\langle 0\rangle$.

\section{REFERENCES}

1. Bloh, A. M. (1965). On a generalization of the concept of Lie algebra. Dokl. AN SSSR, 165, No. 3, pp. 471473.

2. Bloh, A. M. (1967). Cartan-Eilenberg homology theory for a generalized class of Lie algebras. Dokl. AN SSSR, 175, No. 8, pp. 824-826.

3. Bloh, A. M. (1971). A certain generalization of the concept of Lie algebra. Algebra and number theory. Uchenye Zapiski Moskov. Gos. Pedagog. Inst., 375, pp. 9-20.

4. Loday, J.-L. (1993). Une version non commutative des algèbre de Lie: les algèbre de Leibniz. Enseign. Math., 39, pp. 269-293.

5. Loday, J.-L. (1998). Cyclic homology. Grundlehren der Mathematischen Wissenschaften, Vol. 301. 2nd ed. Berlin: Springer.

6. Kirichinko, V. V., Kurdachenko, L. A., Pypka, A. A. \& Subbotin, I. Ya. (2017). The some aspects of Leibniz algebra theory. Algebra Discrete Math., 24, No. 1, pp. 1-33.

7. Albeverio, S., Omirov, B. A. \& Rakhimov, I. S. (2005). Varieties of nilpotent complex Leibniz algebras of dimension less than five. Commun. Algebra, 33, No. 5, pp. 1575-1585.

8. Ayupov, S. A. \& Omirov, B. A. (1999). On 3-dimensional Leibniz algebras. Uzbek. Math. Zh., 1, pp. 9-14.

9. Casas, J. M., Insua, M. A., Ladra, M. \& Ladra, S. (2012). An algorithm for the classification of 3-dimensional complex Leibniz algebras. Linear Algebra Appl., 436, No. 9, pp. 3747-3756.

10. Demir, I., Misra, K. C. \& Stitzinger, E. (2014). On some structures of Leibniz algebras. Recent advances in representation theory, quantum groups, algebraic geometry, and related topics. Contemporary mathematics, Vol. 623, pp. 41-54.

11. Kurdachenko, L. A., Otal, J. \& Pypka, A. A. (2016). Relationships between factors of canonical central series of Leibniz algebras. Eur. J. Math., 2, No. 2, pp. 565-577.

12. Barnes, D. (2011). Some theorems on Leibniz algebras. Commun. Algebra, 39, No. 7, pp. 2463-2472.

Received 05.03.2018 


\section{B.С. Ящук}

Дніпровський національний університет ім. Олеся Гончара

E-mail: Viktoriia.Yashchuk@i.ua

\section{АЛГЕБРИ ЛЕЙБНІЦА ВИМІРНОСТІ 3 НАД СКІНЧЕННИМИ ПОЛЯМИ}

Першим кроком у вивченні всіх типів алгебр є опис таких алгебр, які мають малі вимірності. На відміну від більш простих випадків одно- і двовимірних алгебр Лейбніца, структури тривимірних алгебр Лейбніца складніші. У роботі розглядається структура алгебр Лейбніца вимірності 3 над скінченним полем. У деяких випадках структура алгебр суттєво залежить від характеристики поля, в інших - від можливості розв'язання конкретних рівнянь у полі і т. п.

Ключові слова: алгебра Лейбніча, ідеал, фактор-алгебра, нільпотентна алгебра Лейбніиа.

\section{В.С.Ящук}

Днипровский национальний университет им. Олеся Гончара

E-mail: Viktoriia.Yashchuk@i.ua

\section{АЛГЕБРЫ ЛЕЙБНИЦА РАЗМЕРНОСТИ 3 НАД КОНЕЧНЫМИ ПОЛЯМИ}

Первым шагом в изучении всех типов алгебр является описание таких алгебр, которые имеют малые размерности. В отличие от более простых случаев одно- и двумерных алгебр Лейбница, структуры трехмерных алгебр Лейбница сложнее. В работе рассматривается структура алгебр Лейбница размерности 3 над конечным полем. В некоторых случаях структура алгебры зависит от характеристики поля, в других - от разрешимости конкретных уравнений в поле и т. п.

Ключевые слова: алгебра Лейбница, идеал, фактор-алгебра, нильпотентная алгебра Лейбница. 